\title{
Pal 13: its moderately extended low density halo and its accretion history
}

\author{
Andrés E. Piatti ${ }^{1,2 \star}$ and José G. Fernández-Trincado ${ }^{3}$ \\ ${ }^{1}$ Consejo Nacional de Investigaciones Científicas y Técnicas, Godoy Cruz 2290, C1425FQB, Buenos Aires, Argentina; \\ 2 Observatorio Astronómico, Universidad Nacional de Córdoba, Laprida 854, 5000, Córdoba, Argentina; \\ ${ }^{3}$ Instituto de Astronomía y Ciencias Planetarias, Universidad de Atacama, Copayapu 485, Copiapó, Chile
}

Received / Accepted

\section{ABSTRACT}

\begin{abstract}
We present results on the basis of Dark Energy Camera Legacy Survey (DECaLS) DR8 astrometric and photometric data sets of the Milky Way globular cluster Pal 13. Because of its relative small size and mass, there has not been yet a general consensus about the existence of extra-tidal structures around it. While some previous results claim for the absence of such features, others have shown that the cluster is under the effects of tidal stripping. From DECaLS $g, r$ magnitudes of stars placed along the cluster Main Sequence in the color-magnitude diagram - previously corrected by interstellar reddening-, we built the cluster stellar density map. The resulting density map shows nearly smooth contours around Pal 13 out to $\approx 1.6$ times the most recent estimate of its Jacobi radius, derived by taking into account its variation along its orbital motion. This outcome favors the presence of stars escaping the cluster, a phenomenon frequently seen in globular clusters that have crossed the Milky Way disc a comparable large number of times. Particularly, the orbital high eccentricity and large inclination angle of this accreted globular cluster could have been responsible for the relatively large amount of cluster mass lost.
\end{abstract}

Key words. Galaxy: globular clusters: general - techniques: photometric - globular clusters: individual: Pal 13.

\section{Introduction}

Extended stellar structures have been observed around a nonnegligible number of Galactic globular clusters (Carballo-Bello et al. 2012). For instance, Correnti et al. (2011) discovered an extended stellar halo surrounding the distant NGC 5694, while Olszewski et al. (2009) found an unprecedented extratidal, azimuthally smooth, halo-like diffuse spatial extension of NGC 1851. A similar structure was also found around 47 Tuc Piatti (2017a). Long tidal tails have been detected in the field of NGC 288 (Shipp et al.2018), NGC 5466 (Belokurov et al.|2006), NGC 7492 (Navarrete et al.2017), Pal 1 (Niederste-Ostholt et al. 2010), Pal 5 (Odenkirchen et al. 2003), Pal 14 (Sollima et al. 2011), Pal 15 (Myeong et al. 2017), $\omega$ Cen (Ibata et al. 2019) and M 5 (Grillmair 2019); and other globular clusters were found to be embedded in a diffuse stellar envelope extending to a radial distance of at least five time the nominal tidal radius, like M 2 (Kuzma et al.2016). From a theoretical point of view, some N-body simulations show that potential escapers (Küpper et al. 2010) or potential observational biases (Balbinot \& Gieles 2018) could contribute to the detection of extended envelopes around globular clusters, among others.

There has been some discussion in the literature about the existence of extra-tidal features around Pal 13. Küpper et al. (2011) performed $N$-body simulations from which they found that the cluster is most likely near to its apogalacticon and therefore appear supervirial and blown-up, so that extra-tidal stars got pushed back into the vicinity of the cluster. Bradford et al. (2011) obtained an outer surface brightness slope shallower than that for

^ e-mail: andres.piatti@unc.edu.ar typical globular clusters, so that at large distance, tidal debris are likely affecting the cluster stellar density profile. According to the authors, they could be an evidence for tidal stripping. Another couple of still not reconciled results are those derived by Kundu et al. (2019) and Yepez et al. (2019), respectively, using Gaia proper motions. While the former concluded on the absence of extra-tidal RR Lyrae stars ripped apart from the cluster due to tidal disruption, the latter found that Gaia proper motions of cluster members show a significant scatter, consistent with an scenario of a cluster being tidally stripped. Siegel et al. (2001) also suggested a significant degree of tidal destruction on the basis of various observational evidence.

It is worth mentioning that the cluster tidal radius has been attained in several studies, resulting in remarkably different values. For instance, Côté et al. (2002) determined a tidal radius of $r_{t}=26^{\prime} \pm 6^{\prime}$ from surface density and surface brightness profiles. The authors mention that details of background subtraction and model-fitting lead to describe Pal 13 as either containing a substantial population of extra-tidal stars, or being considerably more spatially extended than previously thought. Later, Bradford et al. (2011) derived a smaller tidal radius of $r_{t}=13.9^{\prime} \pm 1.5^{\prime}$ from a maximum likelihood method applied to all stars in a color-magnitude diagram (CMD) selection window, while Sollima et al. (2018) using the same data set and a different analysis method adopted $r_{l i m}=11.2^{\prime}$. More recently, Baumgardt et al. (2019) estimated $r_{t}=4.94^{\prime}$ by comparing the cluster density profile to a large suite of direct $N$-body star cluster simulations. Note that the value tabulated in Harris (1996, 2010 Edition)'s catalogue is $r_{t}=2.19^{\prime}$.

Massari et al. (2019) have associated the origin of the globular cluster Pal 13 to the Sequoia dwarf galaxy, which took part of 
an early substantial accretion event that contributed to the formation of the Milky Way stellar halo (Myeong et al. 2019), which is also supported by the identification of extended tidal debris with globular cluster abundance like patterns in the inner halo (e.g. Fernández-Trincado et al. 2019). Other six globular clusters have also been associated to the same progenitor, namely: FSR 1758, IC 4499, NGC 3201, 5466, 6101 and 7006. As far as we are aware, 4 of them with previous studies of their external structures have been found to have extra-tidal features : FSR 1758: Barbá et al. (2019); NGC 3202: Kunder et al. (2014); NGC 5466, and NGC 7006: Jordi \& Grebel (2010).

A recent example of this phenomenon are globular clusters associated to Gaia Enceladus (also known as Gaia Sausage), a major accretion event that built the stellar halo of the Milky Way (Belokurov et al. 2018). Indeed, 9 out of 10 associated globular clusters have studies of their outer regions and all of them show some of the above mentioned signatures. For instance, NGC 1851, 1904, 2298 and 2808 exhibit tidal tails (Carballo-Bello et al. 2018); extra-tidal features have been found in NGC 362 (Vanderbeke et al. 2015), NGC 7089 (Kuzma et al. 2016) and NGC 6779 (Piatti \& Carballo-Bello 2019), while Carballo-Bello et al. (2012) mapped the extended envelopes of NGC 1261 and 6864, respectively.

We here exploit the Dark Energy Camera Legacy Survey (DECaLS, Dey et al. 2019) in order to address the issue about the existence of extra-tidal features around Pal 13. Section 2 describes the retrieved data sets, while in Section 3 we deal with the construction of the intrinsic cluster stellar density map. The corresponding analysis in the context of the cluster origin and kinematics is explained in Section 4. Finally, Section 5 summarizes the main outcomes of this work.

\section{Data handing}

We downloaded all the information available in the DECaLS.DR8 ${ }^{1}$ catalogue for an area of $2^{\circ} \times 2^{\circ}$ centered on Pal 13 . The retrieved catalogue contains 306454 sources, for which astrometric and photometric data are provided homogeneously. As a quality check, we only kept in the subsequent analysis those sources with morphological model 'PSF' (stellar point sources), which resulted to have errors in the PSF $g$ magnitude and $g-r$ color less than 0.04 and $0.07 \mathrm{mag}$, respectively. These upper limits for the photometric errors allow us to deal with a photometry completeness nearly 100 per cent at $g \leq 23.0$ mag for the outer cluster regions, where crowding effects are negligible (Dey et al. 2019).

The most recent values of the Galactic extinction are also available on the DR8 catalogues, from which we built the reddening map shown in Fig. 1 . We computed the average for two circular regions, delineated in the figure by red circles with radii of $6^{\prime}$ and $30^{\prime}$, respectively. We obtained $\langle E(B-V)>$ $=0.114 \pm 0.004 \mathrm{mag}$ and $0.117 \pm 0.022 \mathrm{mag}$ for the smallest and largest circles, respectively. These values show that differential reddening should not mislead our interpretation of the stellar density distribution around Pal 13.

The intrinsic (reddening corrected) cluster CMD is depicted in Fig. 2 (left panel) for all the measured stars distributed within the smallest red circle (see Fig. 1). Note that intrinsic $g_{o}$ magnitudes and $(g-r)_{o}$ colors are available in the DR8 catalogues. As can be seen, the main cluster features -including some dispersion from field star contamination- are clearly distinguished. For comparison purposes, we built a reference intrinsic star field

\footnotetext{
1 http://legacysurvey.org/dr8/
}

CMD from stars distributed within an annular region located far away from the cluster and with an area equal to that of the smallest red circle (right panel of Fig. 2). It reveals that the star field contamination in the cluster CMD should not hamper a reliable tracing of the stellar density map in the outer cluster regions.

\section{Stellar density map}

It is well-known that, because of two-body relaxation, the less massive a star, the outer the cluster regions they can reach. Hence, they are candidates to cross the Jacobi radius and thus to populate the cluster extra-tidal regions (Carballo-Bello et al. 2012). In previous studies of the external regions of the globular cluster NGC 288 (Piatti 2018a) and NGC 6779 (Piatti \& Carballo-Bello 2019), we used a strip along the cluster Main Sequence (MS), from underneath its MS turnoff down to 2 mag, to map the distribution of their stellar populations beyond their tidal radii. Those stars are low mass enough as to have been subject of tidal effects, so that we expect that their counterparts in Pal 13 should also be, particularly because Pal 13 is less massive than NGC 288 and NGC 6779 (Baumgardt et al. 2019).

Following the above recipe, we defined the area in the cluster CMD shown by the red contour in Fig. 2(left panel) to build the respective stellar density map. It comprises as many cluster MS stars as possible and minimizes the field star contamination (see right panel of Fig. 2). Nevertheless, we applied a procedure to get rid of field stars that fall inside the defined strip. The method was devised by Piatti \& Bica (2012) and used satisfactorily for cleaning CMDs of star clusters projected towards crowded star fields (e.g., Piatti $2017 \mathrm{~b}$ c a and references therein) and affected by differential reddening (e.g., Piatti 2018b; Piatti et al. 2018. and reference therein). It relies on an accurate representation of the star field CMD in terms of its stellar density, luminosity function and color distribution. This is done by considering the position of each field star in the cluster CMD and by subtracting the closest star in the cluster CMD to that field star. In doing this, we considered the uncertainties in magnitudes and colors by repeating the procedure hundred of times with magnitudes and colors varying within their respective errors. For the designed MS strip, photometric errors increase from $\approx 0.01$ mag up to 0.04 in $g_{o}$ and from $\approx 0.01 \mathrm{mag}$ up to $0.07 \mathrm{mag}$ in $(g-r)_{o}$ for the range $g_{o}=$ 21- 23 mag.

As for the reference star field, we chose an annular region centered on the cluster, located relatively far away from it, but not to far as to lose the star field characteristics in the direction toward the cluster. The chosen annulus is meant to embrace an appropriate collection of any possible star field population and reddening variation around Pal 13. The cleaned circular cluster area $\left(r<30^{\prime}\right)$ and the star field annular region are of equal size. The latter is illustrated with black circles in Fig. 1 .

From the resulting cleaned cluster CMD, we built the stellar density map for those stars spread within the boundaries of the defined MS strip and located inside a circle of radius $r=30^{\prime}$. We used a kernel density estimator (KDE) technique. Particularly, we employed the KDE routine within AstroML (Vanderplas et al. 2012). We superimposed a grid of $400 \times 400$ squared cells to the area of interest and used a range of values for the KDE bandwidth, from $0.3^{\prime}$ up to $3^{\prime}$ in step of $0.3^{\prime}$, in order to apply the KDE to each generated cell. KDE also estimated an optimal bandwidth of $1.5^{\prime}$, which means that we resolved structural details larger than $\approx 1 / 7$ of $r_{t}=4.94^{\prime}$ (Baumgardt et al. 2019). We also estimated the background level using the stars distributed within the annular region defined above (black circles in Fig. 1). We divided such an annulus in 16 adjacent sectors of 


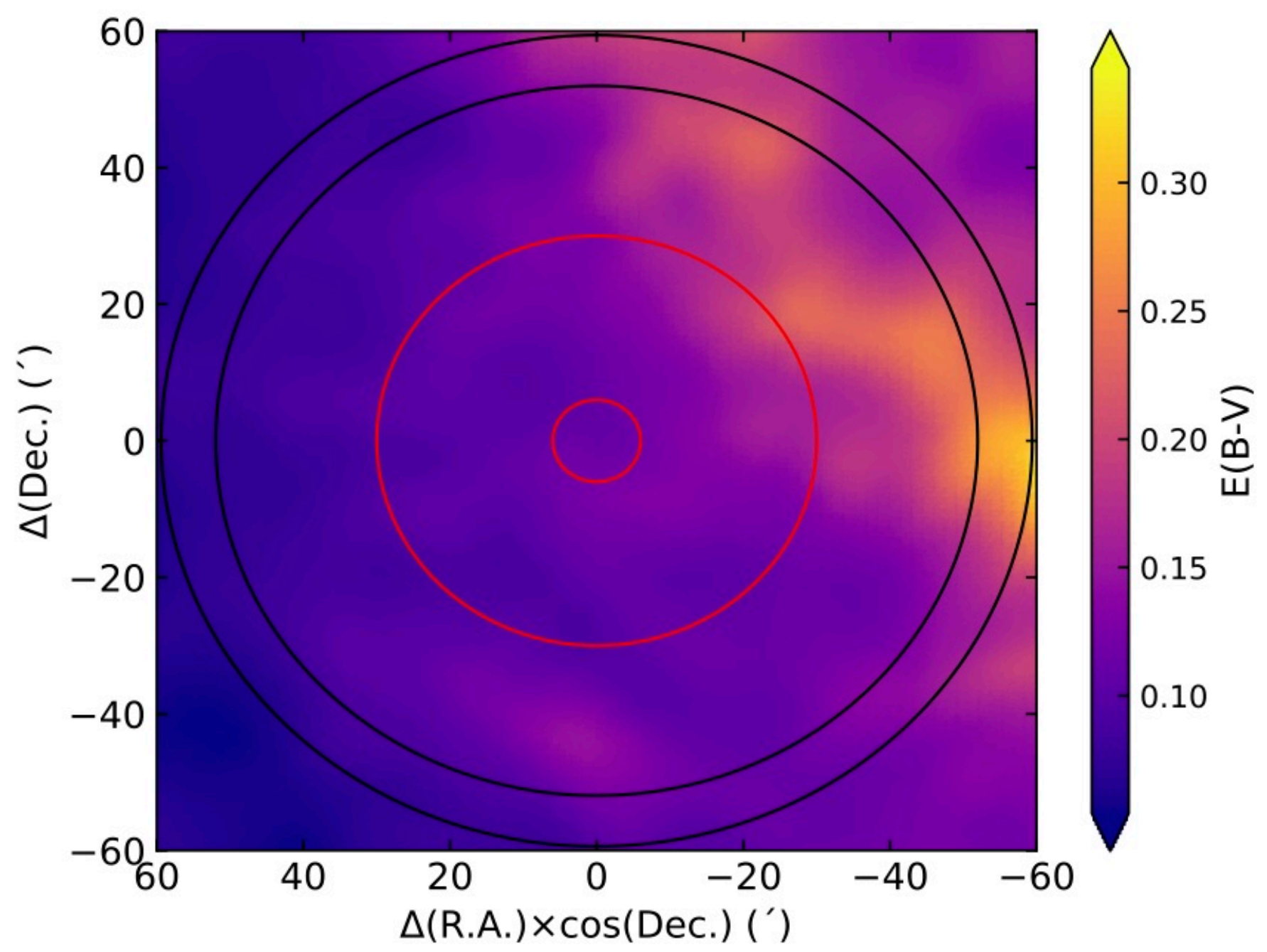

Fig. 1. Reddening map across the field of Pal 13. The red circles are of $6^{\prime}$ and $30^{\prime}$ in radius. The outer black annular region is of the same size as the largest red circular region.

$22.5^{\circ}$ wide, where we counted the number of stars. We rotated such an array of sectors by $11.25^{\circ}$ and repeated the star counting. Finally, we derived the mean value in the 32 defined sectors, which turned out to be $0.037 \mathrm{stars} / \operatorname{arcmin}^{2}$. As for the standard deviation, we performed 1000 Monte Carlos realizations using the stars located beyond $10^{\prime}$ from the cluster center, which were rotated randomly (one different angle for each star) and then the density map recomputed. The resulting standard deviation of all the generated density maps turned out to be $0.011 \mathrm{stars} / \mathrm{arcmin}^{2}$.

The resulting observed and field star cleaned stellar density maps are depicted in the left and right panels of Fig. 3, respectively. The color scale represent the standard deviations over the mean value in the field, i.e., $\eta=($ signal -0.037$) / 0.011$. We have painted white stellar densities higher than $10 \eta$ in order to highlight the cluster less dense stellar structures. Every point used to generate the field star cleaned density map were also employed to build the cluster stellar radial profile shown in Fig. 4

We additionally considered different star field regions distributed beyond $40^{\prime}$ from the cluster centre and applied the same cleaning procedure for their respective MS strips using in all the executions the reference star field as for Pal 13. We found that the resulting cleaned stellar density maps do not contain any visible structure above $1 \eta$, which means that the residuals of the cleaning technique resulted to be negligible.

\section{Analysis and discussion}

The observed cluster MS strip stellar density map would seem to suggest the existence of a concentration of some number of stars from $\approx 5^{\prime}$ out to $\approx 10^{\prime}$ from the cluster centre, following an azimuthally irregular pattern (see Fig. 3, left panel). The lack of detection of farther stellar densities that could be visibly associated to Pal 13, call our attention on the previously derived larger $r_{t}$ values : $14^{\prime}<r_{t}<26^{\prime}$ : Côté et al. (2002); Bradford et al. (2011). To this regard, we recall that field star contamination and uncorrected stellar density profiles from incompleteness of star counts in the inner cluster regions because of crowding could lead to fit nonphysical motivated King (1962) core and tidal radii.

Here we used a field star cleaning procedure that has turned out to be effective in getting rid of such a contamination and thus highlighting the intrinsic extra-tidal features of globular clusters projected on crowded fields or affected by differential reddening (e.g. Piatti \& Carballo-Bello 2019). Particularly, Pal 13 would not seem to be projected on either a field affected by a significant differential reddening (see Section 2) or on a crowded star field (see Fig. 2, right panel). From this point of view, the cluster is an easy target for our purposes, besides the effectiveness of the cleaning method even in more complicated cluster fields. 

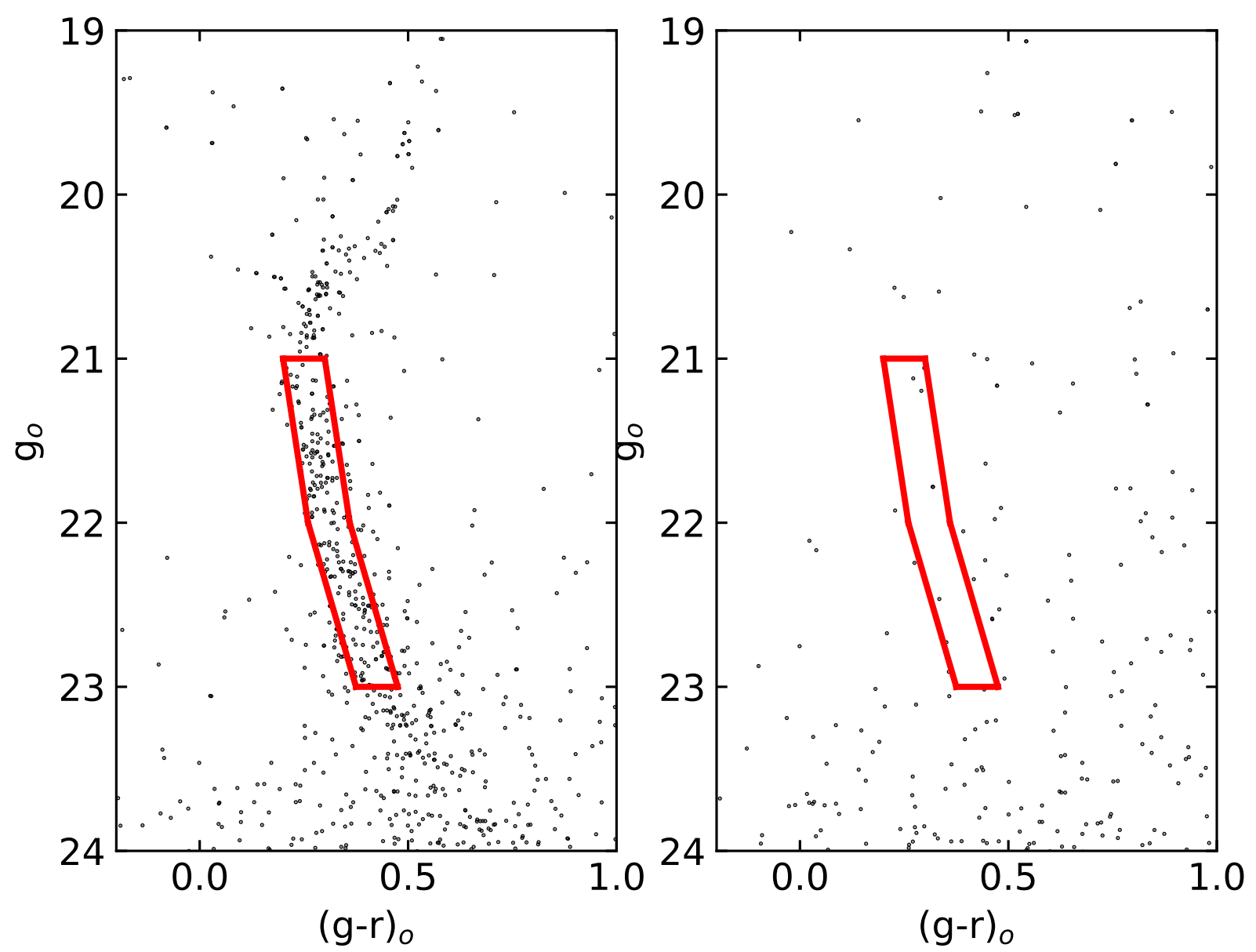

Fig. 2. CMDs of stars in the field of Pal 13 ( $r<6^{\prime}$; left panel) and in an annular region of similar area centered on the cluster with outer radius of $60^{\prime}$ (right panel). The region along the cluster MS used to perform star counts is delineated with red contour lines.

The resulting cleaned stellar density map shown in Fig. 3 (right panel) exhibits a more rounded shape than the observed one (Fig. 3, left panel), with some scattered stellar debris. The figure also shows an excess of stars above $3 \eta$ beyond the adopted limiting radius $r_{t}=4.94^{\prime}$ ( $\equiv$ Jacobi' $\mathrm{s}$ radius, black circle in Fig. 3), obtained by Baumgardt et al. (2019) using eq. 8 in Webb et al. (2013). We refer the reader to Piatti et al. (2019) for a discussion on the uncertainties of the globular cluster parameters derived by Baumgardt et al. (2019) from the integration of their orbital motions. Particularly, they estimated a typical error of the Jacobi radius of $1.2^{\prime}$ at the Galactocentric distance of Pal 13. Fig. 4 also illustrates the numbers given above.

We further analyzed the possibility of tidal deformations across the cluster stellar density map, in the sense that preferential orientation toward the Galactic center and along the direction of the cluster's orbit are expected in the innermost and outermost parts, respectively (Montuori et al.2007). We followed to recipe applied by Sollima et al. (2011) based on counts of cluster MS strip stars in alternate pairs of $90^{\circ}$ wide circular sectors located at a given distance from the cluster center and oriented at a position angle (PA) in opposite directions. Then, we computed the ratio $R(P A)=\left(N_{c}^{A} N_{f}^{B}\right) /\left(N_{c}^{A} N_{f}^{B}\right)$, where $A$ and $B$ are the pair of alternate sectors, and $c$ and $f$ refer to the cluster MS strip and a CMD field rectangle defined by $21<g_{o}(\mathrm{mag})<23$ and $1.5<(g-r)_{o}$ (mag) $<1.7$. In order to assess the statistical significance of our results, we performed 1000 Monte Carlos realizations using the same number of measured stars distributed randomly in PA and them obtained the mean and standard deviations of those independent executions. Fig. 5 depicts the resulting curves. As can be seen, there is no noticeable tidal deformations across Pal 13. For completeness purposes we included in Fig. 3 (right panel) the directions toward the Galactic center and of the cluster's motion. About the globular cluster itself, extra-tidal extensions towards the S-W and N-E directions are marginally present around the cluster. Pal 13 has recently undergone a gravitational shock ( 0.6 Gyr according to GravPot16), so that it is very likely that the extra-tidal extensions visible in Fig. 3 (right panel) correspond to a very recent disk-shocking, with the extra-tidal material aligned toward the tidal directions.

Some recent works on the extended structures of globular clusters have found that at the outer regions the stellar density is $\propto r^{-\alpha}$, with $\alpha$ between 1 and 2 (e.g., Olszewski et al. 2009. Piatti 2017a). If we assumed a power law decrease of the stellar density for the outermost cluster region with slope $\alpha=1$ and 2 , we would find that Pal 13 vanishes down to $3 \eta$ level at $\approx 59^{\prime}$ and $17^{\prime}$, respectively. The power law profiles for $\alpha=1$ and 2 are shown in Fig. 4 with blue and red lines, respectively. Note that the calculation of $r_{t}$ involves the cluster over the course of its 

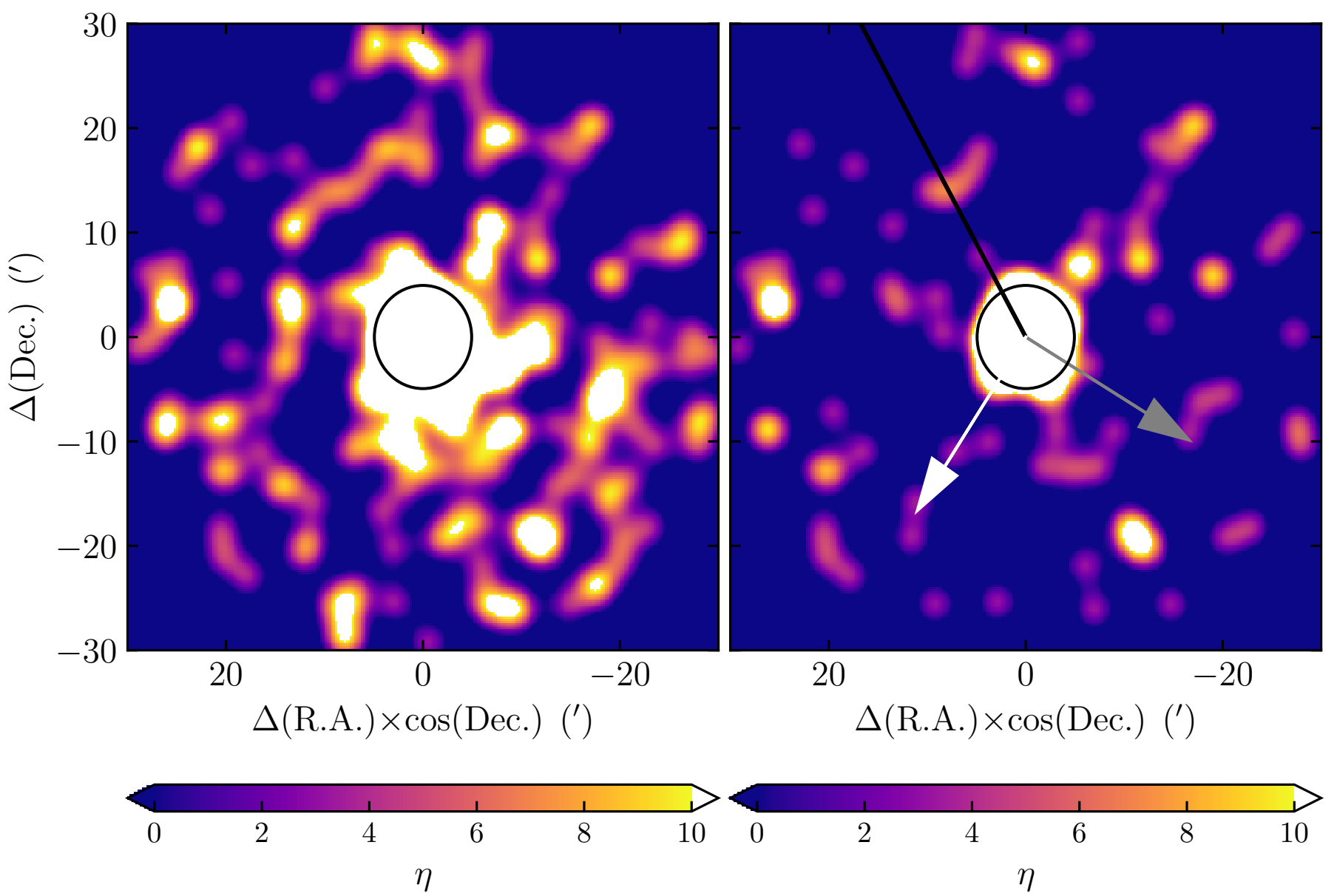

Fig. 3. Observed (left panel) and field star cleaned (right panel) stellar density maps, built from stars that occupy the MS strip defined in Fig. 2 The black circle centered on the cluster indicates the assumed tidal radius Baumgardt et al. (4.94' 2019). The different arrows indicate the directions of the cluster proper motion (gray) and of the Galactic center (white). The black line represents the cluster orbit computed with GravPot 16.

orbits, so that it varies from the perigalactic $(9.04 \pm 1.74 \mathrm{kpc})$ up to the apogalactic $(67.48 \pm 12.5 \mathrm{kpc})$ distances between $2.6^{\prime}$ and $7.2^{\prime}$, respectively (Piatti et al. 2019). This outcome suggests that its present extra-tidal population reaches $\approx 1.4 \times r_{t}$.

Recently, Piatti (2019) used the Baumgardt et al. (2019)'s catalogues to investigate the kinematics of the Milky Way globular clusters. His results show that outer globular clusters are prone to have more eccentric orbits (high eccentricity) than globular clusters moving in the Milky Way disk, regardless the direction of their motions (prograde or retrograde orbits). Their orbits also preferentially have large inclination angles. As far as accreted globular clusters are concerned, they show radial orbits independently of their position in the Galaxy. Globular clusters whose orbits have inclination angles $\lesssim 50^{\circ}$ have experienced several disk crossings compared to those moving along more circular orbits in the disk at a similar Galactocentric distance. Therefore, they have lost more mass (Gnedin et al. 1999; Webb et al. 2014). Piatti et al. (2019) suggested that the lack of outer clusters rotating in nearly circular orbits (and also low inclination angles) could be due to their accreted origin, while the lack of such clusters in the inner Milky Way regions could be due to disruption.

According to Baumgardt et al. (2019), Pal 13 describes a retrograde orbital motion $\left(V_{\phi}=-73.52 \mathrm{~km} / \mathrm{s}\right)$, has an eccentricity of $0.76 \pm 0.05$ and and orbital inclination of $112.26^{\circ} \pm 6.26^{\circ}$. The ratio of the radial to total space velocity is 0.86 and the semimajor axis $a$ (average between the perigalactic and apogalactic distances) is $38.26 \pm 5.50 \mathrm{kpc}$, respectively. The ratio of the cluster mass lost due to Milky Way tidal disruption to the total initial cluster mass computed by Baumgardt et al. (2019) is 0.36, assuming that the cluster lost half of its initial mass via stellar evolution. All these features could favor an accreted origin for Pal 13. Indeed, Koposov et al. (2019) mention this cluster, alongside other 6 ones as clusters located at $\left|\phi_{2}\right|<7^{\circ}$ of the Orphan stream's great circle, whose progenitor could be a dwarf galaxy. On the other hand, Massari et al. (2019) using kinematics and chemical abundance information assigned individual progenitors to most of the known Milky Way globular clusters, the Sequoia dwarf galaxy being that associated one to Pal 13. Koch \& Côté (2019) also discuss this issue.

Küpper et al. (2011) and Balbinot \& Gieles (2018) mention that the cluster is close to its apogalactic distance. Küpper et al. (2011) claimed that because of its position, the cluster should have experienced an expansion that could have encompassed any extra-tidal structure into the expanded body. Balbinot \& Gieles (2018) found that cluster tails are more densely packed at apogalacticon, so that the cluster should be in the best condition of tails (extra-tidal features) observability. From Baumgardt et al. (2019), Pal 13 is currently at a Galactocentric distance of $25.92 \mathrm{kpc}$, so that is 1.5 times farther from its apogalactic distance than from its perigalactic one (see also the cluster's orbit in Yepez et al. (2019)). At that position, the cluster exhibits a moderate extended halo of low density. Furthermore, according 


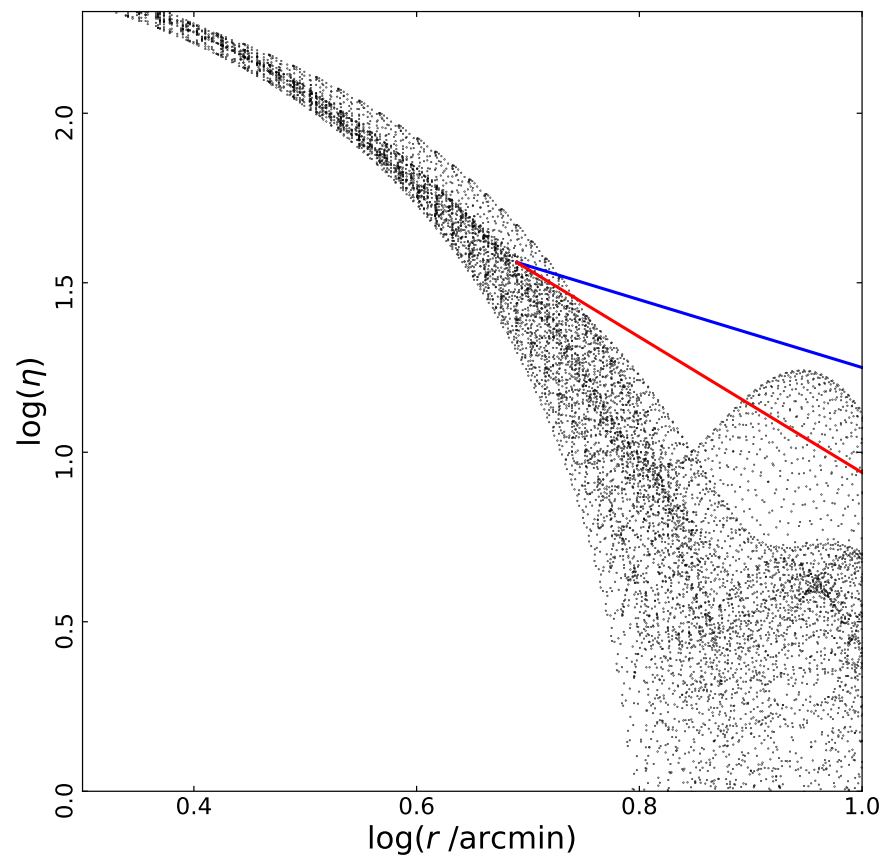

Fig. 4. Standard deviations over the mean value in the field as a function of the distance from the cluster center traced with every point generated from the KDE technique (see text for details). The blue and red lines correspond to a power law with $\alpha=1$ and 2, respectively.

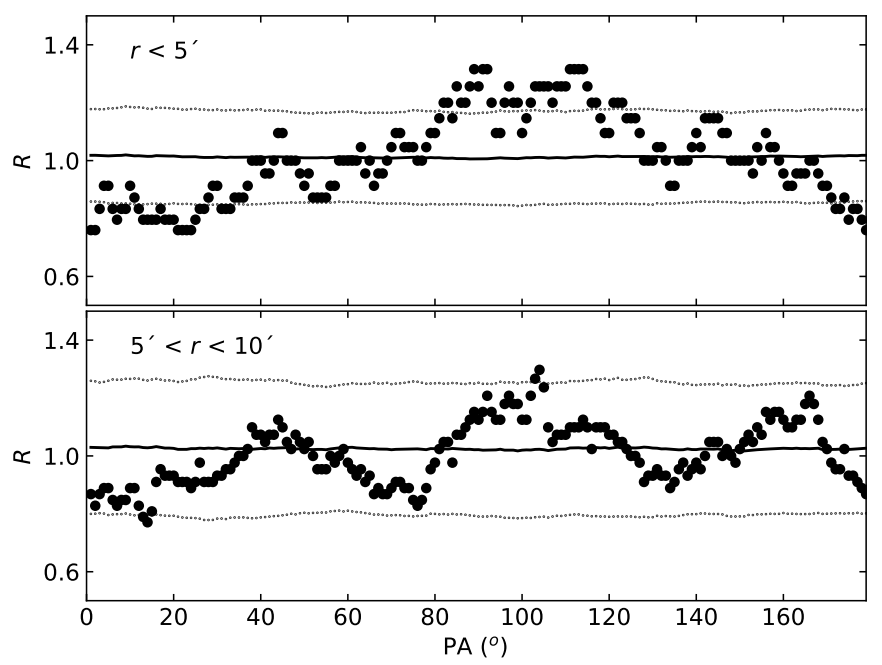

Fig. 5. $R$ versus PA relationship obtained from star counts in the star field cleaned MS strip (large filled circles). Solid and dotted lines represent the resulting mean and dispersion of the Monte Carlo simulations, respectively.

to the above estimated $r_{t}$ values, the extra-tidal corona would also be observed even at the apogalactic distance.

The presence of extra-tidal features in Pal 13 could be alternatively related with the relatively large estimated ratio of mass lost by tidal disrution (0.36). Hamren et al. (2013) showed that low-mass globular cluster may have lost a considerable amount of mass as compared to those more massive clusters. Fig. 6(left panel) shows the ratio of the cluster mass disrupted by tidal effects to the total cluster mass as a function of the semi-major axis for the entire sample of globular clusters in Baumgardt et al. (2019). Points have been colored according to the initial mass as indicated by the horizontal color bar. Pal 13 is shown with a large starred symbol. As can be seen, there is not a clear correlation between the mass lost and the initial cluster mass for clusters at a similar semi-major axis as Pal 13, as suggested by Hamren et al. (2013). However, if we reproduce that plot using the eccentricity as the colored scale variable, a clearer correlation arises, in the sense that the higher the orbital eccentricity of a globular cluster with similar semi-major axis as Pal 13, the higher the mass lost by tidal disruption. This outcome is in the line of the recent $\mathrm{Pi}$ atti et al. (2019)'s results. Their figure 1 shows that the cluster eccentricity plays a role in the amount of cluster mass lost by tidal disruption, because of the more numerous disk crossing experienced respect to clusters with more rounded orbital motions. Likewise, their figure 4 reflects that present-day cluster limiting radii, for clusters with similar semi-major axes, are smaller for those that have lost larger amount of mass due to tidal effects, which we corroborate for Pal 13. These results are fully compatible with Pal 13 being relatively compact and at the same time exhibits extra-tidal features.

\section{Conclusions}

In this work we took advantage of the DECaLS DR8 data sets to address the issue about the existence of extra-tidal structures around Pal 13, one of the relatively compact and less massive known Milky Way globular clusters. Precisely because of its size, some previous works have concluded that the cluster does not extent beyond its Jacobi radius, and that the farthest lowmass members are quite distant from that boundary. Such a result has not been conclusive, since complementary works based on Gaia DR2 proper motions or surface stellar density profiles have found evidence about the cluster is being tidally stripped. Additionally, there has been no consensus about its actual dimension, as judged by the wide range of values derived for its tidal radius.

As far as we are aware, the Panoramic Survey Telescope and Rapid response System (Pan-STARRS PS1, Chambers et al. 2016) and DECaLS are the only present publicly available astrometric and photometric surveys covering a wide area around Pal 13, making them valuable tools for studying the cluster extended structures. We decided to use DELCaLS DR8 because of its comparative better performance in terms of limiting magnitude, photometric errors and completeness. Such a data set was employed to build a stellar density map of stars distributed along the cluster MS, down to 2 mag below the MS turnoff.

At first glance, the cluster stellar density map shows nearly smooth contours around the outer cluster regions, that seem to reach out to $\approx 1.6$ its limiting radius. Here, we adopted the most recent value of $r_{t}=4.94^{\prime}$ derived by Baumgardt et al. (2019) using line-of-sight velocity and proper motion velocity dispersion profiles fitted from a grid of dedicated $N$-body simulations. This outcome favours the presence of stars escaping the cluster, a phenomenon frequently seen in globular clusters that have crossed the Milky Way disc a large number of times (Piatti et al. 2019). Indeed, the high eccentricity and large inclination angle of the Pal 13's orbital motion help us to speculate on that possibility. Furthermore, because of its retrograde direction of motion, Pal 13 has been suggested to have an accreted origin, particularly associated to the Sequoia progenitor when its age and overall metallicity are also considered (Massari et al. 2019). Others six globular clusters could have been formed in the same dwarf galaxy, and four of them with studies of their external regions also have extra-tidal features.

Acknowledgements. We thank the referee for the thorough reading of the manuscript and timely suggestions to improve it. We thank Eugene Vasiliev 

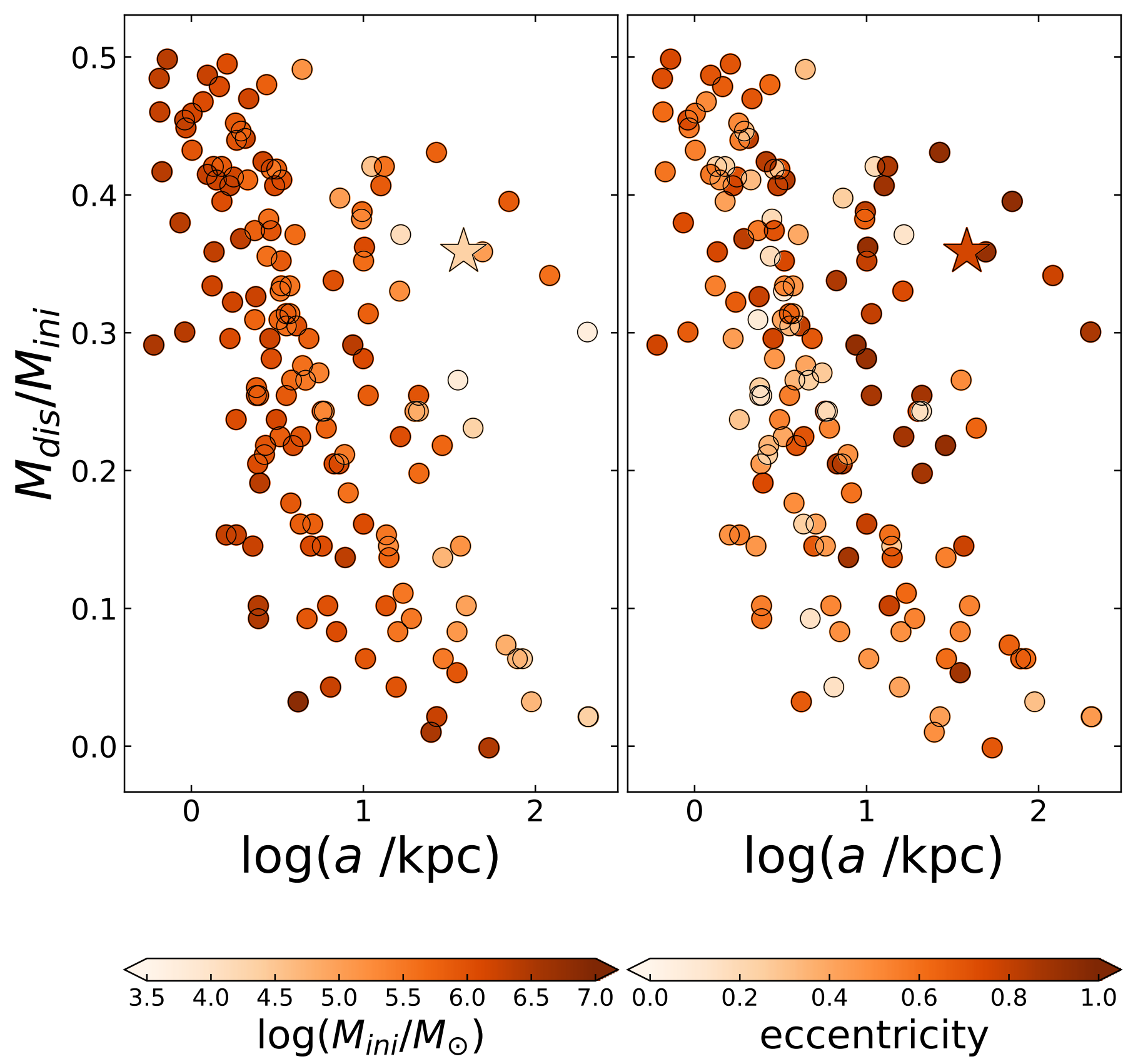

Fig. 6. Relationship between the ratio of the cluster mass lost by tidal effects to the initial cluster mass as a function of the cluster orbit semimajor axis for the entire sample of Milky Way globular clusters in the Baumgardt et al. (2019)'s catalogues. colored symbols are according to the respective horizontal color bars. Pal 13 is represented by a large starred symbol.

for letting us know about the DECaLS public data sets for Pal 13. The Legacy Surveys consist of three individual and complementary projects: the Dark Energy Camera Legacy Survey (DECaLS; NOAO Proposal ID \# 2014B-0404; PIs: David Schlegel and Arjun Dey), the Beijing-Arizona Sky Survey (BASS; NOAO Proposal ID \# 2015A-0801; PIs: Zhou Xu and Xiaohui Fan), and the Mayall zband Legacy Survey (MzLS; NOAO Proposal ID \# 2016A-0453; PI: Arjun Dey). DECaLS, BASS and MzLS together include data obtained, respectively, at the Blanco telescope, Cerro Tololo Inter-American Observatory, National Optical Astronomy Observatory (NOAO); the Bok telescope, Steward Observatory, University of Arizona; and the Mayall telescope, Kitt Peak National Observatory, NOAO. The Legacy Surveys project is honored to be permitted to conduct astronomical research on Iolkam Du'ag (Kitt Peak), a mountain with particular significance to the Tohono O'odham Nation. NOAO is operated by the Association of Universities for Research in Astronomy (AURA) under a cooperative agreement with the National Science Foundation. This project used data obtained with the Dark Energy Camera (DECam), which was constructed by the Dark Energy Survey (DES) collaboration. Funding for the DES Projects has been provided by the U.S. Department of Energy, the U.S. National Science Foundation, the Ministry of Science and Education of Spain, the Science and Technology Facilities Council of the United Kingdom, the Higher Education Funding Council for England, the National Center for Supercomputing Applications at the University of Illinois at Urbana-Champaign, the Kavli Institute of Cosmological Physics at the University of Chicago, Center for Cosmology and Astro-Particle Physics at the Ohio State University, the Mitchell Institute for Fundamental Physics and Astronomy at Texas A\&M University, Financiadora de Estudos e Projetos, Fundacao Carlos Chagas Filho de Amparo, Financiadora de Estudos e Projetos, Fundacao Carlos Chagas Filho de Amparo a Pesquisa do Estado do Rio de Janeiro, Conselho Nacional de Desenvolvimento Cientifico e Tecnologico and the Ministerio da Cien- 
cia, Tecnologia e Inovacao, the Deutsche Forschungsgemeinschaft and the Collaborating Institutions in the Dark Energy Survey. The Collaborating Institutions are Argonne National Laboratory, the University of California at Santa Cruz, the University of Cambridge, Centro de Investigaciones Energeticas, Medioambientales y Tecnologicas-Madrid, the University of Chicago, University College London, the DES-Brazil Consortium, the University of Edinburgh, the Eidgenossische Technische Hochschule (ETH) Zurich, Fermi National Accelerator Laboratory, the University of Illinois at Urbana-Champaign, the Institut de Ciencies de l'Espai (IEEC/CSIC), the Institut de Fisica d'Altes Energies, Lawrence Berkeley National Laboratory, the Ludwig-Maximilians Universitat Munchen and the associated Excellence Cluster Universe, the University of Michigan, the National Optical Astronomy Observatory, the University of Nottingham, the Ohio State University, the University of Pennsylvania, the University of Portsmouth, SLAC National Accelerator Laboratory, Stanford University, the University of Sussex, and Texas A\&M University. BASS is a key project of the Telescope Access Program (TAP), which has been funded by the National Astronomical Observatories of China, the Chinese Academy of Sciences (the Strategic Priority Research Program "The Emergence of Cosmological Structures" Grant \# XDB09000000), and the Special Fund for Astronomy from the Ministry of Finance. The BASS is also supported by the External Cooperation Program of Chinese Academy of Sciences (Grant \# 114A11KYSB20160057), and Chinese National Natural Science Foundation (Grant \# 11433005). The Legacy Survey team makes use of data products from the Near-Earth Object Wide-field Infrared Survey Explorer (NEOWISE), which is a project of the Jet Propulsion Laboratory/California Institute of Technology. NEOWISE is funded by the National Aeronautics and Space Administration. The Legacy Surveys imaging of the DESI footprint is supported by the Director, Office of Science, Office of High Energy Physics of the U.S. Department of Energy under Contract No. DE-AC02-05CH1123, by the National Energy Research Scientific Computing Center, a DOE Office of Science User Facility under the same contract; and by the U.S. National Science Foundation, Division of Astronomical Sciences under Contract No. AST-0950945 to NOAO. A.E.P. acknowledge support from the Ministerio de Ciencia, Tecnología e Innovación Productiva (MINCyT) through grant PICT-201-0030. J.G.F-T is supported by FONDECYT No. 3180210 and Becas Iberoamérica Investigador 2019, banco Santarder Chile.

\section{References}

Balbinot, E. \& Gieles, M. 2018, MNRAS, 474, 2479

Barbá, R. H., Minniti, D., Geisler, D., et al. 2019, ApJ, 870, L24

Baumgardt, H., Hilker, M., Sollima, A., \& Bellini, A. 2019, MNRAS, 482, 5138

Belokurov, V., Erkal, D., Evans, N. W., Koposov, S. E., \& Deason, A. J. 2018, MNRAS, 478, 611

Belokurov, V., Evans, N. W., Irwin, M. J., Hewett, P. C., \& Wilkinson, M. I. 2006, ApJ, 637, L29

Bradford, J. D., Geha, M., Muñoz, R. R., et al. 2011, ApJ, 743, 167

Carballo-Bello, J. A., Gieles, M., Sollima, A., et al. 2012, MNRAS, 419, 14

Carballo-Bello, J. A., Martínez-Delgado, D., Navarrete, C., et al. 2018, MNRAS, 474,683

Chambers, K. C., Magnier, E. A., Metcalfe, N., et al. 2016, ArXiv e-prints [arXiv: 1612.05560$]$

Correnti, M., Bellazzini, M., Dalessandro, E., et al. 2011, MNRAS, 417, 2411

Côté, P., Djorgovski, S. G., Meylan, G., Castro, S., \& McCarthy, J. K. 2002, ApJ, 574,783

Dey, A., Schlegel, D. J., Lang, D., et al. 2019, AJ, 157, 168

Fernández-Trincado, J. G., Beers, T. C., Placco, V. M., et al. 2019, ApJ, 886, L8

Gnedin, O. Y., Lee, H. M., \& Ostriker, J. P. 1999, ApJ, 522, 935

Grillmair, C. J. 2019, arXiv e-prints, arXiv:1909.05927

Hamren, K. M., Smith, G. H., Guhathakurta, P., et al. 2013, AJ, 146, 116

Harris, W. E. 1996, AJ, 112, 1487

Ibata, R. A., Bellazzini, M., Malhan, K., Martin, N., \& Bianchini, P. 2019, Nature Astronomy, 3, 667

Jordi, K. \& Grebel, E. K. 2010, A\&A, 522, A71

King, I. 1962, AJ, 67, 471

Koch, A. \& Côté, P. 2019, arXiv e-prints, arXiv:1910.13506

Koposov, S. E., Belokurov, V., Li, T. S., et al. 2019, MNRAS, 485, 4726

Kunder, A., Bono, G., Piffl, T., et al. 2014, A\&A, 572, A30

Kundu, R., Minniti, D., \& Singh, H. P. 2019, MNRAS, 483, 1737

Küpper, A. H. W., Kroupa, P., Baumgardt, H., \& Heggie, D. C. 2010, MNRAS, 401,105

Küpper, A. H. W., Mieske, S., \& Kroupa, P. 2011, MNRAS, 413, 863

Kuzma, P. B., Da Costa, G. S., Mackey, A. D., \& Roderick, T. A. 2016, MNRAS, 461, 3639

Massari, D., Koppelman, H. H., \& Helmi, A. 2019, A\&A, 630, L4

Montuori, M., Capuzzo-Dolcetta, R., Di Matteo, P., Lepinette, A., \& Miocchi, P. 2007, ApJ, 659, 1212

Myeong, G. C., Jerjen, H., Mackey, D., \& Da Costa, G. S. 2017, ApJ, 840, L25
Myeong, G. C., Vasiliev, E., Iorio, G., Evans, N. W., \& Belokurov, V. 2019 MNRAS, 488, 1235

Navarrete, C., Belokurov, V., \& Koposov, S. E. 2017, ApJ, 841, L23

Niederste-Ostholt, M., Belokurov, V., Evans, N. W., et al. 2010, MNRAS, 408 L66

Odenkirchen, M., Grebel, E. K., Dehnen, W., et al. 2003, AJ, 126, 2385

Olszewski, E. W., Saha, A., Knezek, P., et al. 2009, AJ, 138, 1570

Piatti, A. E. 2017a, ApJ, 846, L10

Piatti, A. E. 2017b, ApJ, 834, L14

Piatti, A. E. 2017c, MNRAS, 465, 2748

Piatti, A. E. 2018a, MNRAS, 473, 492

Piatti, A. E. 2018b, MNRAS, 477, 2164

Piatti, A. E. 2019, ApJ, 882, 98

Piatti, A. E. \& Bica, E. 2012, MNRAS, 425, 3085

Piatti, A. E. \& Carballo-Bello, J. A. 2019, MNRAS, 485, 1029

Piatti, A. E., Cole, A. A., \& Emptage, B. 2018, MNRAS, 473, 105

Piatti, A. E., Webb, J. J., \& Carlberg, R. G. 2019, MNRAS, 489, 4367

Shipp, N., Drlica-Wagner, A., Balbinot, E., et al. 2018, ApJ, 862, 114

Siegel, M. H., Majewski, S. R., Cudworth, K. M., \& Takamiya, M. 2001, AJ, 121,935

Sollima, A., Martínez Delgado, D., Muñoz, R. R., et al. 2018, MNRAS, 476, 4814

Sollima, A., Martínez-Delgado, D., Valls-Gabaud, D., \& Peñarrubia, J. 2011, ApJ, 726, 47

Vanderbeke, J., De Propris, R., De Rijcke, S., et al. 2015, MNRAS, 450, 2692

Vanderplas, J., Connolly, A., Ivezić, Ž., \& Gray, A. 2012, in Conference on Intelligent Data Understanding (CIDU), $47-54$

Webb, J. J., Harris, W. E., Sills, A., \& Hurley, J. R. 2013, ApJ, 764, 124

Webb, J. J., Sills, A., Harris, W. E., \& Hurley, J. R. 2014, MNRAS, 445, 1048

Yepez, M. A., Arellano Ferro, A., Schröder, K. P., et al. 2019, New A, 71, 1

Article number, page 8 of 8 